\title{
A completeness proof for an infinitary tense-logic
}

by

\author{
GÖRAN SUNDHOLM
}

(University of Uppsala)

In his forthcoming examination of G. H. von Wright's tense-logic [4], Krister Segerberg studies certain infinitary extensions of the original tense-logic created by von Wright. For one of these extensions the completeness problem turned out to be harder than was expected at first sight. ${ }^{1}$ This paper is devoted to a proof of a completeness theorem for the extension in question, called $W 1$ by Segerberg.

We use a countable language of ordinary propositional logic supplied with two modal operators: $\bigcirc$ ("tomorrow") and $\square$ ("always"). The relevant semantics for tense-logic based on this language uses the frame $\mathfrak{N}=\left\langle N,{ }^{\prime}, \leq\right\rangle$, where the successorrelation is the accessibility-relation for $\bigcirc$ and $\leq$ for $\square$, i.e., the formula $\circ(A)$ is true at the point $n \in N$ iff $A$ is true at $n+1$, and the formula $\square A$ is true at $n \in N$ iff for all $k \geq n A$ is true at $k$. We assume that the reader is familiar with ordinary Kripkesemantics for modal languages and, in particular, that he understands what it means that " $\mathfrak{M}$ is a model on the frame $\mathfrak{N}$ ". We shall use $O^{k}(A)$ as a shorthand for

$$
\underbrace{\circ(\circ(\ldots \circ}_{k \text { times }}(A) \ldots)) \text {. }
$$

\footnotetext{
1 Professor Segerberg's original proof idea turned out to be incomplete in that it used the Lindenbaum lemma, as is usual in canonical model proofs. Because of the infinitary rules we do not have immediate access to the Lindenbaum lemma, and I then undertook to prove the lemma using the proof theoretic machinery hinted at the end of the paper. However, Professor Dag Prawitz pointed out an embarassing error in my argument for which I am very grateful. I also want to express my gratitude to Professor Segerberg for much encouragement.
} 
Let $\Sigma$ be a set of formulae from our language. $\Sigma$ is said to have a model on $\mathfrak{N}$ if there is a model $\mathfrak{N}$ on $\mathfrak{N}$ such that for some $n \in N$ it holds $\mathfrak{N} \vDash_{n} A$, for every $A$ in $\Sigma$.

The main part of Professor Segerberg's paper is spent on a proof that if a finite $\Sigma$ is consistent in von Wright's tense logic, then it has a model on $\mathfrak{N}$. Since the rules of von Wright's logic are finitary, a set is consistent iff all its finite subsets are. As Segerberg observes, the set $\theta=\{\neg \square p\} \cup\left\{O^{n}(p): n \in N\right\}$ is consistent in von Wright's logic, for every finite subset thereof has a model on $\mathfrak{N}$ and is thus consistent. $\theta$ itself, however, has no model on $\mathfrak{N}$. In order to improve on this fact Segerberg introduces an infinitary extension $W 1$ of von Wright's logic. $W 1$ is given by a Prawitz-type natural deduction system, and we assume some familiarity with, e.g., [3].

For every $n \in N$ :

$$
\wedge \mathrm{E}(n) \frac{\bigcirc^{n}\left(A_{1} \wedge A_{2}\right)}{\bigcirc^{n}\left(A_{i}\right)} \quad(i=1,2) \quad \wedge \mathrm{I}(n) \frac{\bigcirc^{n}\left(A_{1}\right) \bigcirc^{n}\left(A_{2}\right)}{\bigcirc^{n}\left(A_{1} \wedge A_{2}\right)}
$$

$$
\begin{aligned}
& {\left[O^{n}\left(A_{1}\right)\right]\left[O^{n}\left(A_{2}\right)\right]} \\
& \vee \mathrm{E}(n) \frac{\mathrm{O}^{n}\left(A_{1} \vee A_{2}\right) \quad B \quad B}{B} \vee \mathrm{I}(n) \frac{O^{n}\left(A_{i}\right)}{O^{n}\left(A_{1} \vee A_{2}\right)}(i=1,2) \\
& {\left[O^{n}(A)\right]} \\
& \rightarrow \mathrm{E}(n) \frac{\bigcirc^{n}(A \rightarrow B) \bigcirc^{n}(A)}{\bigcirc^{n}(B)} \\
& \rightarrow \mathrm{I}(n) \frac{\mathrm{O}^{n}(B)}{\mathrm{O}^{n}(A \rightarrow B)} \\
& {[\neg A] \quad[\neg A]} \\
& \text { [A] } \\
& \text { [A] } \\
& \neg \mathrm{E}(n) \frac{\neg \mathrm{O}^{n}(B) \quad \neg^{n}(\neg B)}{A} \\
& \neg \mathrm{I}(n) \frac{\mathrm{O}^{n}(B) \quad \mathrm{O}^{n}(\neg B)}{\neg A} \\
& \square \mathrm{E}(n) \frac{\mathrm{O}^{n}(\square A)}{\mathrm{O}^{n+k}(A)} k=0,1,2, \ldots \\
& \square \mathrm{I}(n) \frac{\left\langle\mathrm{O}^{n+k}(A)\right\rangle_{k \in N}}{\mathrm{O}^{n}(\square A)}
\end{aligned}
$$

We will prove the following theorem, first stated by Segerberg in $\S 5$ of [4]. 
Theorem. If $\Sigma$ is consistent in $W 1$ it has a model on $\Re$.

The proof is of the Henkin-type and is modelled on Feferman's completeness-proof for $\mathrm{L}_{\omega_{1} \omega}$ in [1]. The crux of the proof is the fundamental

Lemma. If $\Sigma$ is a consistent set in $W 1$, then so is $\Sigma \cup\left\{O^{n+k}(A) \rightarrow\right.$ $\left.\mathrm{O}^{n}(\square A)\right\}$, for some $k \in N$.

Proof. Assume not. Then $\Sigma \cup\left\{O^{n+k}(A) \rightarrow \bigcirc^{n}(\square A)\right\}$ is inconsistent for each $k$. Hence, for each $k \in N, \Sigma \vdash \neg\left(O^{n+k}(A) \rightarrow O^{n}(\square A)\right)$, hence $\Sigma \vdash \bigcirc^{n+k}(A) \wedge \neg \bigcirc^{n}(\square A)$, hence $\Sigma \vdash \bigcirc^{n+k}(A)$ and $\Sigma \vdash \neg O^{n}(\square A)$, and thus $\Sigma \vdash \bigcirc^{n}(\square A)$ and $\Sigma \vdash \neg \bigcirc^{n}(\square A)$, by rule $\square \mathrm{I}(n)$, which contradicts the consistency of $\Sigma$.

Let $\Sigma$ be a consistent set in $W 1$ and $\left\langle A_{0}, A_{1}, \ldots\right\rangle$ an enumeration of our language. We define

$$
\begin{aligned}
& \Sigma_{0}=\Sigma, \\
& \Sigma_{2 n+1}=\left\{\begin{array}{l}
\Sigma_{2 n} \cup\left\{A_{n}\right\} \text { if this is consistent, } \\
\Sigma_{2 n} \cup\left\{\neg A_{n}\right\} \text { otherwise, }
\end{array}\right. \\
& \Sigma_{2 n+2}=\left\{\begin{array}{l}
\Sigma_{2 n+1} \cup\left\{O^{m+k}(B) \rightarrow \bigcirc^{m}(\square B)\right\} \text { if } A_{n}=\bigcirc^{m}(\square B), \\
\Sigma_{2 n+1} \text { otherwise. }
\end{array}\right.
\end{aligned}
$$

In step $2 n+2, k$ should be chosen as small as possible while preserving consistency according to the Lemma. We observe that by construction each $\Sigma_{n}$ is consistent in $W 1$. Let $\Delta=\cup \Sigma_{n}$. This set $\Delta$ has all the properties needed for a canonical model proof.

(i) For all $A, A \in \Delta$ or $\neg A \in \Delta$, but not both. Proof. $A=A_{n}$, for some $n$. In step $2 n+1$ either $A$ or $\neg A$ is added. If both are in $\Delta$ then they are in some $\Sigma_{n}$, but this contradicts consistency.

(ii) $\circ^{n}(\neg A) \in \Delta$ iff $\circ^{n}(A) \notin \Delta$. Proof. If $\circ^{n}(\neg A) \in \Delta$ and $\circ^{n}(A) \in \Delta$ then both are in some $\Sigma_{m}$, which would then be inconsistent by two applications of rule $\neg \mathrm{I}(n)$. If $\circ^{n}(A) \notin \Delta$ then $\neg^{n}(A) \in \Delta$. Assume $\bigcirc^{n}(\neg A) \notin \Delta$. Then $\neg \bigcirc^{n}(\neg A) \in \Delta$. But then some $\Sigma_{k}$ would be inconsistent by rule $\neg \mathrm{E}(n)$. 
(iii) $\circ^{n}(B \wedge C) \in \Delta$ iff $\circ^{n}(B) \in \Delta$ and $\circ^{n}(C) \in \Delta$. Proof. Assume $O^{n}(B), O^{n}(C)$ and $\neg \bigcirc^{n}(B \wedge C)$ all belong to $\Delta$. The some $\Sigma_{k}$ is inconsistent by rules $\wedge \mathrm{I}(n)$ and $\neg \mathrm{E}(0)$. The converse is similar. (iv) $\bigcirc^{n}(A \vee B) \in \Delta$ iff $\bigcirc^{n}(A) \in \Delta$ or $\bigcirc^{n}(B) \in \Delta$. Proof. Similar to (iii).

(v) $\circ^{n}(A \rightarrow B) \in \Delta$ iff $\circ^{n}(A) \in \Delta$ implies $\circ^{n}(B) \in \Delta$. Proof. There is no difficulty in showing the statement from left to right. Assume for other way that the right hand is true but $\neg^{n}(A \rightarrow B) \in \Delta$. Two cases: (a) $\circ^{n}(B) \in \Delta$. Then $\neg \bigcirc^{n}(A \rightarrow B)$, $\mathrm{O}^{n}(B)$ both belong to some $\Sigma_{k}$, which would be inconsistent by rules $\rightarrow \mathrm{I}(n)$ and $\neg \mathrm{E}(0)$. (b) $\bigcirc^{n}(B) \notin \Delta$. Then $\bigcirc^{n}(A) \notin \Delta$ and thus $\neg^{n}(A) \in \Delta$. Hence $\neg \bigcirc^{n}(A)$ and $\neg^{n}(A \rightarrow B)$ belong to some $\Sigma_{k}$. Then $\Sigma_{k}$, ${ }^{n}(A) \vdash \mathrm{O}^{n}(B)$ by rule $\neg \mathrm{E}(0)$ and thus $\Sigma_{k} \vdash \mathrm{O}^{n}(A \rightarrow B)$, which contradicts the consistency.

(vi) $\bigcirc^{n}(\square A) \in \Delta$ iff $\bigcirc^{n+k}(A) \in \Delta$, all $k \in N$. Proof. Left to right is again easy. For the other way we use that by construction there is a formula $\bigcirc^{n+k}(A) \rightarrow \bigcirc^{n}(\square A)$ in $\Delta$. If the right side is true and the left false, then by using the formula above some $\Sigma_{m}$ would be inconsistent.

The model is now defined by

$$
\mathfrak{N} \vDash_{n} p \text { iff } \circ^{n}(p) \in \Delta \text {, for propositional letters } p \text {. }
$$

Using (i)-(vi) it requires no effort to prove that

$$
\mathfrak{M} \vDash_{n} A \text { iff } \circ^{n}(A) \in \Delta \text {, for all } A \text {. }
$$

Hence by putting $n=0$ we get the required model on $\Re$, not only for $\Sigma$ but also for $\Delta$. Note that the countability of the language is used essentially in the proof.

It should be remarked here that by dropping $\vee$ and $\neg$ from our language and adding absurdity $\perp$ as a primitive with the rules

$$
\begin{array}{r}
{[A \rightarrow \perp]} \\
\bigcirc^{n}(\perp) \frac{\bigcirc^{n}(\perp)}{A}
\end{array}
$$


then one will get a system that is easily seen to be mutually interpretable with $W 1$. For this system one proves without much effort a normalization theorem along the lines of [3] and [2]. The remarkable ease with which the natural deduction methods work for W1, and especially for the modified version hinted at above, should be credited to the great analogy between $W 1$ and Peano arithmetic with the omega-rule for which it is well-known that a smooth proof theory exists.

\section{References}

[1] Solomon Fefrerman. "Lectures on proof theory". In Proceedings of the Summer School of Logic, Leeds, 1967, Lecture notes in mthematics, vol. 70, pp. 1-107 (Berlin: Springer, 1968)

[2] PrR Martin-Lór. "Infinite terms and a system of natural deduction." Compositio mathematica, vol. 24 (1972), pp. 93-103.

[3] DAG PRAwitz. Natural deduction: A proof-theoretical study. (Stockholm: Almqvist \& Wiksell, 1965)

[4] Krister Segrrberg. "von Wright's tense logic." Forthcoming in The philosophy of G. H. von Wright, to be edited by P. A. Schilpp.

Received on January 30, 1976. 